\title{
NOAA GENERAL MODELING MEETING AND FAIR BRINGS TOGETHER ITS MODELING ENTERPRISE
}

\author{
Annarita Mariotti, John Dunne, Jason Link, Hendrik Tolman, Kevin Garrett, \\ Derrick Snowden, and Timothy Schneider
}

$\mathrm{T}$ he 2018 NOAA Modeling Meeting and Fair ${ }^{1}$ brought together experts across many different modeling disciplines to share lessons learned, identify opportunities for synergy, and build closer working relationships across different domains of modeling expertise that exist across NOAA Line Offices and their external partners. This ambitious and unprecedented NOAA event invited a diverse group of participants with wide-ranging interests and objectives. The theme for this year's event was "interdisciplinary modeling and partnerships," with the main goal of enhancing modeling communication

AFFILIATIONS: MARIOTTI-NOAA/Office of Oceanic and Atmospheric Research/Climate Program Office, Silver Spring, Maryland; DunNe-NOAA/Office of Oceanic and Atmospheric Research/Geophysical Fluid Dynamics Laboratory, Princeton, New Jersey; LINK—NOAA/National Marine Fisheries Service, Silver Spring, Maryland; Tolman AND SCHNeIder-NOAA/National Weather Service/Office of Science and Technology Integration, Silver Spring, Maryland; GARRETT—NOAA/NESDIS/Center for Satellite Applications and Research, College Park, Maryland; SNOWDEN-NOAA/National Ocean Service/Integrated Ocean Observing System, Silver Spring, Maryland

CORRESPONDING AUTHOR: Annarita Mariotti, annarita.mariotti@noaa.gov

DOI:10.|I75/BAMS-D-|8-03|8.|

In final form 6 December 2018

For information regarding reuse of this content and general copyright information, consult the AMS Copyright Policy.

\section{NOAA GENERAL MODELING MEETING AND FAIR \\ WHAT: Nearly 200 scientists and managers from government, academia, and private industry convened for this inaugural event to learn about unified modeling across the National Oceanic and Atmospheric Administration (NOAA) for weather, climate, fisheries, oceans, and coasts across all relevant disciplines, share ideas on how to advance activities, and network with other modeling professionals. \\ WHEN: 10-12 September 2018 \\ WHERE: NOAA Center for Weather and Climate Prediction, College Park, MD}

and networking across disciplines and organizations involved in NOAA modeling to promote unified modeling (Fig. 1). Representatives across all NOAA Line Offices, partner federal agencies, and academic institutions participated in keynote presentations, informal networking events, modeling tutorials, and the Modeling Fair, which included over 60 exhibits from the NOAA modeling enterprise.

Keynote presentations offered overviews of NOAA's vision for modeling activities with a focus on

\footnotetext{
${ }^{1}$ See www.star.nesdis.noaa.gov/ngmmf. This meeting was proposed and organized by the NOAA Research Council's Unified Modeling Committee's Information Exchange Working Group.
} 
interdisciplinary modeling, and related gaps and opportunities across its various Line Offices. At the strategic level, there was an emphasis on the need for a modeling infrastructure that allows external community participation in exploratory research and development, the opportunities provided by public-private partnerships and community modeling, and the need to focus on attracting talented individuals to NOAA modeling careers. NOAA Unified Modeling was discussed as an approach to optimize investments as part of a common framework, not to be mistaken with a "one model" approach. NOAA Line Office presentations described, among other things, how modeling for fisheries applications is increasingly integrated to include physical, ecological, socio-cultural, and economic factors so as to address practical needs; modeling for coasts now spans issues from sea level rise and storm surge to healthy habitats and navigation; and coupled models strive to harness a variety of Earth data, both in situ and from satellites, to best address NOAA's environmental applications. Also highlighted were the increasing emphasis on community modeling to improve weather forecasting as part of a unified forecasting framework and the broad array of NOAA's research activities that develop and apply models to understand and simulate the complexity of the Earth system and improve NOAA service capabilities. Additional presentations provided more specific examples of interdisciplinary modeling at NOAA and in the external community including ecological modeling of harmful algae blooms, Earth system modeling for climate applications at the National Center for Atmospheric Research (NCAR), modeling of processes in the Arctic region by the Department of Energy, and modeling of land processes and data integration by the National Aeronautics and Space Administration (NASA).

The Fair was a key feature of this event. It offered a unique cross-cut view of NOAA's modeling enterprise and its broad interdisciplinarity, and was a great opportunity to directly connect with the scientists and managers involved in NOAA's mission. Participants explored the often interactive exhibits, discussed ongoing work, and established new collaborations for model development or applications. Participants also voted on awards for exhibits of exemplary interdisciplinary modeling activities and efforts to portray modeling via attractive visualizations. During the Fair, modeling tutorials offered participants opportunities to learn in-depth NOAA modeling topics of high interest across multiple disciplines, including NOAA community models such as the new weather model dynamical core Finite-Volume Cubed-Sphere (FV3) and the Modular Ocean Model (MOM6);

\section{NOAA General Modeling MeEting AND FAIR}

20IB THEME: "INTERDISCIPLINARY MODELINg AND PARTNERSHIPS"

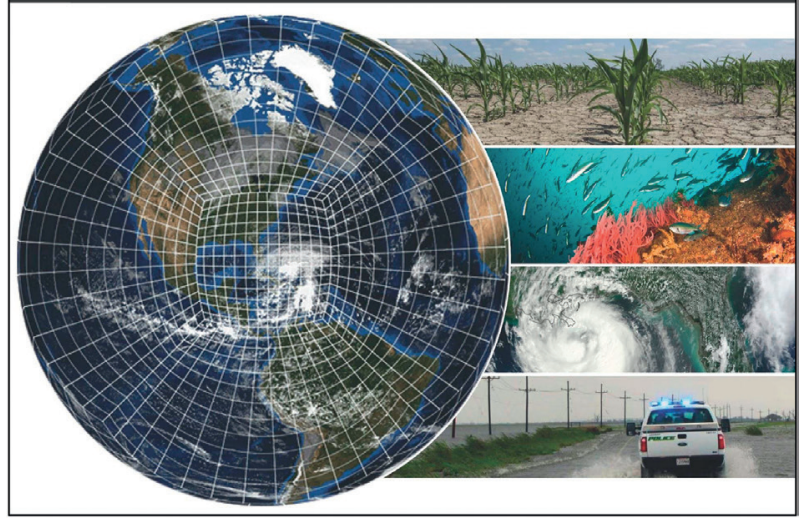

FIG. I. The banner of the 2018 Modeling Meeting and Fair, illustrating the broad interdisciplinarity of NOAA's modeling applications.

basic facts about how NOAA approaches modeling (e.g., coupled modeling for climate and ecosystem modeling for fisheries); and software that is being applied to help advance NOAA modeling capabilities, such as Python and the Joint Effort for Data assimilation Integration (JEDI).

The Meeting and Fair included purposeful opportunities for participants to explore career advancement, especially for early career scientists, and advantages and opportunities to enhance diversity and inclusion. The meeting featured early career scientists in "Lightning Round" talks on their research and an evening "Early Career Scientists" event that allowed for networking and career-oriented discussions with senior NOAA staff. The meeting also included a dynamic session with discussion of ideas on how to capitalize on and expand diversity and inclusion in the NOAA modeling community.

Discussion sessions were held throughout the meeting, with the last day focused on meeting outcomes and future NOAA-wide community engagement. Participants emphasized the need to move toward an increasingly collaborative NOAA modeling culture that builds on purposeful investments in the modeling workforce, including training, a proactive career reward system, sharing of best modeling practices, external community engagement, and partnerships critical to fostering innovation. Meeting discussions also highlighted several factors that could help enhance NOAA modeling. These included optimal use of observations, high-performance computing availability and access, an efficient modeling 
infrastructure, and a framework for model coupling with more integration across NOAA modeling disciplines. Participants highlighted the value of enhanced communication across modeling disciplines and organizations, and ultimately the value to NOAA of better integrating physical, biological, chemical, and socioeconomic modeling. Overall, participants were optimistic about emerging NOAA modeling capabilities resulting from efforts to better integrate the NOAA modeling enterprise by bridging across disciplines, NOAA's internal and external modeling, and research and operations. Participants expressed a desire for continued NOAA modeling community engagement in efforts such as the NOAA General Modeling Meeting and Fair to help accelerate model development and the integration of disciplines and applications across NOAA and the broad community it interfaces with. 